\title{
A Review on Various contrast enhancement scheme for Dark Images
}

\author{
Anubha Prajapati ${ }^{1}$, Monika Agrawal ${ }^{2}$ \\ ${ }^{1}$ (Computer Science and Engineering, OIST, Bhopal , India) \\ ${ }^{2}$ (Computer Science and Engineering, OIST, Bhopal , India)
}

\begin{abstract}
Contrast enhancement technique is used to enhance the perception of the image or scene. This enhancement controls the brightness difference between objects and their backgrounds. It also used as brightness preservation of image. Numerous contrast enhancement techniques are offered by various researchers for the betterment of the quality of images like contrast enhancement of HDR, DCT, DWT, Filtering etc. the common problem with image enhancement is difficult to achieve such images. Noise is an unwanted element of system that creates problem or interference. Contrast enhancement uses scaling to remove internal noise of dark images using DCT technique. Here in this paper we are presenting some techniques used for contrast enhancement of image.
\end{abstract}

Keywords: Contrast Enhancement, Internal Noise, Stochastic Resonance, Noise Reduction, Discrete Cosine Transform.

\section{Introduction}

Contrast enhancement is a way to enrich the quality of images. It uses with numerous techniques to add glory in image enhancement. It majorly deals with the brightness of background of any image best suited for background brightness preservation or enhancement. Contrast enhancements are typically performed as a contrast stretch followed by a tonal enhancement. Although these could both are performed in single step. A contrast stretch improves the brightness differences uniformly across the dynamic range of the image. In such scenario tonal enhancements improve the brightness differences in the dark shadow, grays mid-tone, or bright highlighted regions at the expense of the brightness differences in the other regions. Stochastic resonance is a method to enhance image using noise rather than hiding it. It just likes removing negativity from negative elements. It is a counter-intuitive phenomenon in a nonlinear system where the noise is essential for optimal system performance [1].

Numerous images are found with varying low intensity values just because of improper illumination and hence required processing before displaying them. There are many techniques that are targeted to betterment of gray level images in the spatial domain [2]. Contrast enhancement technique can be further classified in to two categories direct and indirect methods. Direct method is based on defining of contrast measure and corrects the image on the basis of contrast measure. While in indirect method uses dynamic range of underutilized regions for improving contrast without defining it. Indirect methods are widely used and also subcategorized [3]. Examples of some indirect methods are homomorphic filtering, histogram modification techniques [4], [5], [6], [7] and transform based techniques [8], [9].

Apart from this sub group another group also subcategorizes and received the most attention due to its straightforward and intuitive implementation qualities. The second subgroup modifies the image through some pixel mapping such that the histogram of the processed image is more spread than that of the original image. Such techniques enhance the image contrast either locally or globally. In global methods single mapping is derived from image is used to enhance the contrast. While in local method each neighborhood pixel is used in the local tone mapping function to enhance the image. Global method cannot be appropriate for local methods [3].

Contrast enhancement using Histogram Equalization is one of the most popular methods for image enhancement due to its simplicity and efficiency. It usually increases the global contrast of the images mostly in cases where the important and useful data of the image is shown by low contrast values. Histogram equalization (HE) is a simple and effective contrast enhancement technique which distributes pixel values uniformly such that enhanced image have linear cumulative histograms [3]. The HE technique is a global operation hence; it does not preserve the image brightness. DWT includes any wavelet transform for which the wavelets are discretely sampled. Some of the mostly used wavelets include Haar Wavelet, Daubechies Wavelets (DB), Symlets (sym). The wavelet transform used here is the Haar transform, since Haar Transform captures not only a notion of the frequency content of the input, by inspecting it at diverse scales, but also chronological content, i.e. the moment at which these frequencies take place. After applying the DWT, the image is subjected to histogram equalization. Sometimes, the parts of the image that contains the useful data are represented by low 
contrast values. Using histogram equalization method the contrast of these areas is enhanced which provides improved image quality [10].

For the illumination images, HE-based brightness preserving methods fail to extract the detail information of the images. Although the DHE is a powerful method for enhancing the low contrast images, it leads to noise amplifying and intensity saturation problems in some cases. The QDHE consists of four processes, namely the histogram partitioning, clipping, gray level range allocation and histogram equalization. Therefore, the QDHE utilizes the median intensity value of the input image histogram in partitioning the histogram. Initially, the histogram of the original image is divided into two sub-histograms. Similarly, the medians from the two partitioned sub-histograms are used as separating points to further divide the two subhistograms into two smaller sub-histograms each. Thus, there are a total of four sub-histograms obtained. Then, the minimum and maximum intensity values of the input histogram are set as the separating points [11].

Based upon qualitative and quantitative analyses the automatic exact histogram specification technique has been found effective in enhancing contrasts of images in comparison to a few existing methods. Quantitative evaluation of contrast enhancement is not trivial, as there don't exist any universally accepted measure of contrast or ideally enhanced images as references. Measures of dispersion (local and global) such as variance, standard deviation and entropy have been used to evaluate contrast enhancement. Quantitative evaluation of contrast enhancement should be based upon appropriate measurement of contrast at all image pixels. Any reasonable measure of contrast should be at least crudely tuned according to the retinal visual system and such a measure would then probably be more credible and universally acceptable. Global contrast enhancement is aimed at increasing the overall contrast of an image. Local contrast enhancement is aimed at increasing contrast in local neighborhoods in images in order to reveal minute details [12].

The satellite image contrast enhancement technique based on DWT and SVD decomposed the input image into the DWT subbands, and, after updating the singular value matrix of the LL subband, it reconstructed the image by using IDWT. There are two significant parts of this scheme. The first one is the use of SVD. As it was mentioned, the singular value matrix obtained by SVD contains the illumination information. Therefore, changing the singular values will directly affect the illumination of the image; hence, the other information in the image will not be changed. The second one is application of DWT. The edges are concentrated in the other subbands (i.e., LH, HL, and HH). Hence, separating the high-frequency subbands and applying the illumination enhancement in the LL subband only will protect the edge information from possible degradation. After reconstructing the final image by using IDWT, the resultant image will not only be enhanced with respect to illumination but also will be sharper [13].

The optimum internal noise is inherent in its DCT coefficients and so that the DCT coefficients can be viewed as image information along with noise. Contrast enhancement via Dynamic Stochastic Resonance applied iteratively in image enhancement using DCT coefficients. It is uses RGB color band to improve the image quality. It is best suited for very dark image with highest gray level less than 100. In this scheme 8X8 block of images are computed. Discrete cosine transform operation was performed in this image blocks to modify localize information. If the entropy of block is less than standard deviation then blocks are subdivided in to 4X4 block size. Noise induced DSR is applied to the discrete cosine transform coefficient to dark area. This scheme is able to enhance the brightness of the dark area effectively [1].

Stochastic resonance is a situation when system experience increased signal to noise ration when input amplitude of input noise rises. If a input signal is less than a sensory detection level SR can improve detection via adding of small noise. It is the technique that is used to detect ice age of the earth earlier. The simple concept of this scheme is that if we add some small amount of noise in to a weak system it enhances the ability to detect that signal. Same concept is used to enhance the quality of image. Here image noise is used against the image to enhance its perception and contrast [14].

The rest of paper is organized as follows. In Section II describes about background of contrast enhancement. In Section III describes about related work of stochastic resonance and contrast enhancement techniques followed by a conclusion in Section IV

\section{Background}

The utility of digital images is very much common for all kinds of display gadgets. Unfortunately, the input images that are captured by these devices are sometimes not really in good brightness and contrast. Therefore, a process known as digital image enhancement is normally required to increase the quality of these low brightness images. The objective of image enhancement techniques is to improve a quality of an image such that enhanced image is better than the original image. Numerous techniques for image improvement have been suggested by various researchers in both spatial and transform domains. Image improvement fabricates a production image that instinctively looks enhanced than the original image by altering the pixel's intensity of the participation image. 


\section{Related Work}

This section describes some related work to image quality improvement, stochastic resonance and contrast enhancement.

\subsection{Non Dynamic Stochastic Resonance based Contrast Enhancement}

A noise-induced dynamic SR-based contrast enhancement technique was investigated by R. K. Jha et al [1]. The iterative process on the noisy coefficients enhances the image energy. This enhancement can be done by making a transition into another state in analogy via inter-well transition of particle in a bi-stable double-well system. It is also observed that the DSR-based enhancement technique surpasses the conventional techniques of image enhancement in both spatial and frequency transform domains [1].

$$
x(n+1)=x(n)+\Delta t\left[\left(a x(n)-b x^{3}(n)\right)+\text { Input }\right]
$$

\subsection{Wavelet Domain Stochastic Resonance based Image Enhancement}

Dynamic Stochastic Resonance based techniques were offered by Chouhan et al [2] to improve the image quality in wavelet domain. Dynamic stochastic resonance is a concept of physics that is used to betterment of the image quality. In this method a DSR-based adaptive algorithm used in discrete-wavelet transform domain for betterment of very dark images. This algorithm optimizes the bi-stable system parameters to maximize performance of an iterative procedure. This algorithm can similarly be used for 2 or higher level DWT but DSR should be applied to approximation coefficients only at one of the levels, preferably at level 1 as that has best resolution. Application of DSR to approximation coefficients affects both brightness and contrast of image in totality. DSR on detail coefficients is conducive to enhancement of edges. If DSR is applied to higher levels, due to successive decrease in resolution, the computational complexity decreases, but best output is obtained on level-1 approximation coefficients. When compared with existing DSR-based technique using DFT, DWT-based technique is observed to give colored output with much less computational complexity [2].

\subsection{Contrast Enhancement using Histogram Equalization}

A general framework for image contrast enhancement was proposed by Arici et al [3] in 2009. They also suggested a minimum complexity scheme that supports video display application. This technique is based on histogram equalization for enhancing the image contrast enhancement. They obtained a visually pleasing enhancement scheme with low-computational complexity and can be easily implemented on FPGAs or ASICs. This technique works well with both video and still images. The stretching parameter should also be adapted with image content. For dark images white stretching can be favoured, while for bright images black stretching can be favoured. The contrast enhanced image obtained by the proposed method is visually more pleasing than HE and WTHE [3].

\subsection{Contrast Enhancementt using IDBPHE}

Brightness preservation called Image Dependent Brightness Preservation Histogram Equalization (IDBPHE) was suggested by P. Rajavel. The reason of image enhancement is to improve the interpretability or perception of information contained in the image for individual viewers, or to make available an improved input for other automated image processing systems. The proposed method includes identifying the bright regions using Discrete Wavelet Transform (DWT). The image obtained after the dynamic histogram equalization of each sub histogram is has the mean brightness that is slightly different than the input image. To remove this difference the normalization process is applied to the output image [10].

In this technique the bright regions of the images are identified using Curvelet Transform and then the histogram of the original image is modified with respect to the histogram of the identified regions. First generation curvelet transform is a multiscale directional transform which has been designed to represent edges and other singularities along curves effectively. It enables directional analysis of images in different scales. Second generation curvelet transform called the discrete curvelet transform (DCvT) has been proposed with less complexity and fast computation. It has two different decompositions namely unique spaced DCvT (UDCvT) and the wrapping DCvT (WDCvT). In UDCvT, the curvelet coefficients are found by irregular sampling the Fourier coefficients of images. In WDCvT, a series of translations and wraparound technique are used [10]

\subsection{Quadrant dynamic histogram equalization}

The QDHE consists of four processes, namely the histogram partitioning, clipping, gray level range allocation and histogram equalization. Therefore, the QDHE utilizes the median intensity value of the input image histogram in partitioning the histogram. The QDHE method obtains almost similar processing time with the RMSHE, the RSIHE and the BHEPL methods, which demonstrate almost similar computation complexity among them. In addition, the proposed QDHE method needs shorter execution time computation compared to the DHE and the BPDHE [11]. 


\subsection{SVD based Contrast Enhancement of Satellite Images}

Demirel et al [13]proposed a new method for satellite image equalization which is an extension of SVE, and it is based on the SVD of an LL subband image obtained by DWT. DWT separates the low contrast satellite image in to various frequency subbands. Here the LL subband concentrates the illumination information so it goes through the SVE process that preserves the high-frequency components. The quality of the visual results indicates that this equalization technique is sharper and brighter than the one achieved by BPDHE, SVE, GHE, and LHE. The resultant image generated by BPDHE is comparable with the image achieved by this method. The visual results on the final image quality show the superiority of the proposed method over the conventional and the state-of-the-art techniques [13].

\subsection{Fingerprint feature extractor using SR}

A fingerprint feature extractor is used instead of a threshold nonlinearity to determine if features can be extracted from a fingerprint image was suggested by C. Ryu et al [14]. Low-quality fingerprint images will act like a weak coherent input. Gaussian noise is used to demonstrate usefulness of SR in fingerprint feature extraction. In this scheme Gaussian noise is used to improve low-quality fingerprint images for those images that were rejected by feature extractors. Gaussian noise also used to enhance Fingerprint feature extraction performance. They show that among ten images nine were rejected by the tradition finger print methods. To overcome this SR based finger print extraction method used. And it efficiently enhance the ten low low-quality fingerprint images out of eleven images [14].

\subsection{Bilateral tone adjustment (BiTA) and Saliency-weighted contrast enhancement (SWCE)}

In 2011 Wei-Ming Ke, Chih-Rung Chen, And Ching-Te Chiu proposed a protocol that combines bilateral tone adjustment (BiTA) and saliency-weighted contrast enhancement (SWCE) methods. It is for the innovative image enhancement framework. BiTA enhances the mid-tone regions and bright and dark regions. They also resented ratio of saliency-weighted relative entropy to noise to evaluate the enhanced quality. This protocol achieves high contrast enhancement with little noise and great image quality [15].

\subsection{Bi-Histogram equalization with neighbourhood metric}

In 2010 Nyamlkhagva Sengee et al [16] proposed a technique Histogram Equalization with Neighbourhood Metric (BHENM). BHENM performs firstly divides large histogram into sub-bins using neighbourhood metrics, the identical intensities of the original or actual image are arranged by neighbouring information and secondly it separates histogram of the original into two sub histograms based on the mean of the histogram of the original image. It produces flatter histogram by equalization of sub-histograms by using refined histogram equalization. BHENM preserves the brightness and enhanced the local contrast of the original image [16].

\subsection{Contrast Enhancement using Local Tone Mapping}

In 2012 by $\mathrm{Ji}$ Won Lee et. al [17] propose a noise reduction method and an adaptive distinguish enhancement for local tone mapping (TM). The proposed local TM algorithm compresses the luminance of high dynamic range (HDR) image and decomposes the compressed luminance of HDR image into multi-scale sub bands using the discrete wavelet transform. The major benefit of this scheme is better result in tone mapping. Image that is produced are rich in colors also. But this scheme is slightly time consuming and color saturation needs more improvement [17].

Internal noise determines external stochastic resonance in visual perception was suggested by Takatsugu Aihara et al [20]. They were interested in the effect of internal noise on external noise-induced sensitization of weak signal detection. It is desirable to adopt an experimental design where external noise and signals interact within the brain. If one uses the single receptor design where external noise and signals are presented to the same eye, external noise and signals first interact in the retina and potentially continue to interact throughout the peripheral visual system. This design guarantees that the random neural activity caused by external visual noise interacts within the central brain with the neural activity caused by visual signals. It is possible just because both noise and signals from the two eyes first converge in early visual cortex. In this they estimated the psychometric function for each noise level in order to estimate the spread (inverse slope), threshold and hit rate which are used for later analysis. Their results suggested that the external SR effects depend on internal noise levels [20]. 
IV. Comparison Of Different Contrast Enhancement Techniques

\begin{tabular}{|l|l|l|l|l|}
\hline Technique used & PSNR & MER & EME & Time \\
\hline Stochastic Resonance & $>50$ & 0.23 & $85 \%$ & Less \\
\hline Histogram Equalization & $>55$ & 0.17 & $85-90 \%$ & Less \\
\hline DCT & $<45$ & 0.58 & $76 \%$ & More \\
\hline SVD & Nearly 48 & 0.49 & $79 \%$ & More \\
\hline IDBPHE & $>52$ & 0.20 & $86 \%$ & Less \\
\hline DWT & $>56$ & 0.14 & $89 \%$ & Less \\
\hline Local Tone Mapping & $>58$ & 0.15 & $86 \%$ & More \\
\hline Clustering based & Nearly 43 & 0.54 & $76 \%$ & More \\
\hline
\end{tabular}

\section{Conclusion}

Generally, images can be grouped into two broad categories: those that have sufficient signal-to-noise ratio (SNR), and those that do not. Images that fall in the first category are typically those with levels of brightness and contrast that are generally classified as good. Conversely, images in the second category tend to be dark with insufficient brightness and contrast. Images in the second category also tend to be the images that benefit the most from image enhancement. Here we are reviewed some techniques that are used to enhance the quality and perception of the images.

\section{References}

[1] Jha, Rajib Kumar, Rajlaxmi Chouhan, Prabir Kumar Biswas, and Kiyoharu Aizawa, Internal noise-induced contrast enhancement of dark images, In $19^{\text {th }}$ IEEE International Conference on Image Processing (ICIP-2012), pp. 973-976, 2012.

[2] Chouhan, Rajlaxmi, C. Pradeep Kumar, Rawnak Kumar, and Rajib Kumar Jha. Contrast Enhancement of Dark Images using Stochastic Resonance in Wavelet Domain. International Journal of Machine Learning and Computing, vol. 2, no. 5, 2012.

[3] Arici, Tarik, Salih Dikbas, and Yucel Altunbasak. A histogram modification framework and its application for image contrast enhancement. IEEE Transactions on Image Processing, vol. 18, no. 9, pp. 1921-1935, 2009.

[4] Z. Y. Chen, B. R. Abidi, D. L. Page, and M. A. Abidi, Gray-level grouping (GLG): An automatic method for optimized image contrast enhancement-Part I: The basic method, IEEE Trans. Image Process., vol. 15, no. 8, pp. 2290-2302, Aug. 2006.

[5] Z. Y. Chen, B. R. Abidi, D. L. Page, and M. A. Abidi, Gray-level grouping (GLG): An automatic method for optimized image contrast enhancement-Part II: The variations IEEE Trans. Image Process., vol. 15, no. 8, pp. 2303-2314, Aug. 2006.

[6] Q. Wang and R. K. Ward Fast image/video contrast enhancement based on weighted thresholded histogram equalization IEEE Trans. Consum. Electron., vol. 53, no. 2, pp. 757-764, May 2007.

[7] C.-C. Sun, S.-J. Ruan, M.-C. Shie, and T.-W. Pai, Dynamic contrast enhancement based on histogram specification IEEE Trans. Consum. Electron., vol. 51, no. 4, pp. 1300-1305, Apr. 2005.

[8] S. Agaian, B. Silver, and K. Panetta , Transform coefficient histogram based image enhancement algorithms using contrast entropy, IEEE Trans. Image Process., vol. 16, no. 3, pp. 741-758, Mar. 2007.

[9] J. Tang, E. Peli, and S. Acton, Image enhancement using a contrast measure in the compressed domain, IEEE Signal Process. Lett., vol.10, no. 10, pp. 289-292, Oct. 2003.

[10] P. Rajavel, Image Dependent Brightness Preserving Histogram Equalization, IEEE Transactions on Consumer Electronics, Vol. 56, No. 2, May 2010.

[11] Ooi, Chen Hee, and Nor Ashidi Mat Isa. Quadrants dynamic histogram equalization for contrast enhancement. IEEE Transactions on Consumer Electronics, vol. 56, no. 4, pp. 2552-2559, 2010.

[12] Sen, Debashis, and Sankar K. Pal. Automatic exact histogram specification for contrast enhancement and visual system based quantitative evaluation. IEEE Transactions on Image Processing,vol 20, no. 5, 1211-1220, 2011.

[13] Demirel, Hasan, Cagri Ozcinar, and Gholamreza Anbarjafari. Satellite image contrast enhancement using discrete wavelet transform and singular value decomposition. IEEE Geoscience and Remote Sensing Letters, vol. 7, no. 2, pp. 333-337, 2010.

[14] Enhancement of feature extraction for low-quality fingerprint images using stochastic resonance

[15] Wei-Ming Ke, Chih-Rung Chen, And Ching-Te Chiu Bita/Swce: Image Enhancement With Bilateral Tone Adjustment and Saliency Weighted Contrast Enhancement IEEE Transactions On Circuits And Systems For Video Technology, Vol. 21, No. 3, March 2011.

[16] Nyamlkhagva Sengee, Altansukh Sengee, and Heung-Kook Choi Image Contrast Enhancement using Bi-Histogram Equalization with Neighborhood Metricsm 2010 IEEE transaction on consumer electronics, Vol 56, no. 4, November 2010

[17] Ji Won Lee, Rae-Hong Park, SoonKeun Chang, Noise Reduction and Adaptive Contrast Enhancement for Local Tone Mapping IEEE Transactions on Consumer Electronics, Vol. 58, No. 2, May 2012.

[18] Chouhan, Rajlaxmi, Rajib Kumar Jha, and Prabir Kumar Biswas. Wavelet-based contrast enhancement of dark images using dynamic stochastic resonance. In Proceedings of the Eighth Indian Conference on Computer Vision, Graphics and Image Processing, article no. 73, ACM, 2012.

[19] Suganya, P., S. Gayathri, and N. Mohanapriya. Survey on Image Enhancement Techniques. International Journal of Computer Applications Technology and Research, vol. 2, no. 5, pp. 623 - 627, 2013.

[20] Aihara, Takatsugu, Keiichi Kitajo, Daichi Nozaki, and Yoshiharu Yamamoto. "Internal noise determines external stochastic resonance in visual perception." Vision research 48, no. 14, pp. 1569-1573, 2008. 